\title{
A global perspective of antibiotic-resistant Listeria monocytogenes prevalence in assorted ready to eat foods: A systematic review
}

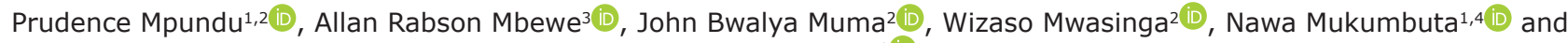 \\ Musso Munyeme ${ }^{2}$ (i)
}

1. Ministry of Health, Levy Mwanawasa Medical University, Lusaka 10101, Zambia; 2. Department of Disease Control, School of Veterinary Medicine, University of Zambia, Lusaka 10101, Zambia; 3. Department of Environmental Health, School of Public Health, University of Zambia, Lusaka, Zambia; 4. Department of Epidemiology and Biostatics, Levy Mwanawasa Medical University, Lusaka, Zambia.

Corresponding author: Prudence Mpundu, e-mail: prudencezimba@gmail.com

Co-authors: ARM: rabmbewe@gmail.com, JBM: jmuma@unza.zm,WM: wizanso14@gmail.com, NM: mktnawa@gmail.com, MM: mussomunyeme@gmail.com

Received: 15-03-2021, Accepted: 20-07-2021, Published online: 26-08-2021

doi: www.doi.org/10.14202/vetworld.2021.2219-2229 How to cite this article: Mpundu P, Mbewe AR, Muma JB, Mwasinga W, Mukumbuta N, Munyeme M (2021) A global perspective of antibiotic-resistant Listeria monocytogenes prevalence in assorted ready to eat foods: A systematic review, Veterinary World, 14(8): 2219-2229.

\begin{abstract}
Background and Aim: Listeria monocytogenes in ready-to-eat (RTE) foods remains consistently under-reported globally. Nevertheless, several independent studies conducted to investigate have elucidated the prevalence and antibiotic resistance profiles of L. monocytogenes in RTE-associated foods and their antibiotic resistance profiles. Given the rapid increase in consumption of RTE foods of both animal and plant origin, it is imperative to know the prevalence deductive data focusing on how much of L. monocytogenes is present in RTE foods, which is critical for food safety managers and retailers to assess the possible risk posed to end-users. In addition, valuable insight and another angle to the depth of the problem, we conducted a systematic review and meta-analysis to synthesize available data regarding the prevalence of $L$. monocytogenes in RTE foods and antibiotic resistance profiles.

Materials and Methods: We conducted a meta-analysis study of L. monocytogenes and antibiotic resistance to clinically relevant antibiotics to determine the extent of $L$. monocytogenes contamination in RTE foods and antibiotic resistance profiles. The primary search terms, also known as keywords used, were restricted to peer-reviewed and review articles, and databases, including Google Scholars, Science-Direct, and Scopus, were searched. The inclusion of articles meeting eligibility criteria published between 2010 and 2020 after title, abstract, and full article screening. Data analysis was performed at multiple stages using quantitative meta-analysis reviews.

Results: L. monocytogenes pooled proportion/prevalence was highest in chicken products determined at (22\%) followed by various but uncategorized RTE foods at $21 \%$. Regarding antibiotic resistance, profiling's highest pooled prevalence resistance was observed in penicillin at $80 \%$ resistance, followed by cephalosporin at $47 \%$.

Conclusion: Within its limitations, this study has attempted to provide insight into the pooled proportion/prevalence of L. monocytogenes in RTE foods and the antibiotic resistance profile at the global level. Determining the proportion/ prevalence of L. monocytogenes in RTE foods across the globe and antibiotic resistance profile is essential for providing quality food and reducing public health problems due to unsuccessful treatment of foodborne illness. This study provides insight into the pooled prevalence of L. monocytogenes in RTE foods and the antibiotic resistance profile. The results of this study partly endeavored to help appropriate authorities strengthen their preventive measures on specific RTE foods that are most likely to be contaminated with L. monocytogenes and antibiotic resistance profiles.
\end{abstract}

Keywords: antibiotic resistance, Listeria monocytogenes, ready-to-eat foods.

\section{Introduction}

Listeria monocytogenes Gram-positive ubiquitous bacterium known to cause listeriosis, recognized as a foodborne pathogen in the early 1980s [1]. It is a known psychotropic microorganism and can survive

\footnotetext{
Copyright: Mpundu, et al. Open Access. This article is distributed under the terms of the Creative Commons Attribution 4.0 International License (http://creativecommons.org/licenses/ by/4.0/), which permits unrestricted use, distribution, and reproduction in any medium, provided you give appropriate credit to the original author(s) and the source, provide a link to the Creative Commons license, and indicate if changes were made. The Creative Commons Public Domain Dedication waiver (http:// creativecommons.org/publicdomain/zero/1.0/) applies to the data made available in this article, unless otherwise stated.
}

in harsh environmental conditions [2]. However, most pathogens, including L. monocytogenes, can be killed by pasteurization temperatures [3]. Unlike most other foodborne pathogens, L. monocytogenes can grow in food with reasonably low moisture content, at refrigeration temperature and high salt concentrations, especially in ready to eat (RTE) foods in contrast with many other foodborne pathogens [4]. It is difficult to measure the global impact of listeriosis infections in assorted foods because the incubation period can be prolonged [5]. Listeriosis is rarely described and is under-reported due to inadequate linkage between the food consumed and the subsequent infection; most 
cases are sporadic, except for food producers who conduct quality assurance activities $[1,6]$.

The World Health Organization (WHO) annual report of 2015 indicated that 600 million people fall ill globally due to foodborne diseases [7]. SouthEast Asia accounts for the highest global burden of foodborne diseases per population among the WHO regions, with global infection and deaths of about 150 million and 175 thousand, respectively [8]. On the other hand, Africa accounts for about 91 million people with foodborne diseases [7]. The United States preliminary report done in 2019 showed increased pathogens such as Listeria transmitted through food. However, the burden remained the same, indicating an impossibility to meeting the set target of "Healthy people 2020" by reducing foodborne illness [9]. RTE chicken samples in United Kingdom between 2016 and 2017 were analyzed, where an association by the authors regarding the source of human listeriosis was established [10]. A report done in the European Union (EU) indicated that despite the application of food safety criteria for L. monocytogenes in RTE foods, no significant decrease from their findings was recorded in human invasive listeriosis [11]. The significant increasing trend of listeriosis was observed during 2011-2015 by the EU member states from 1516 cases in 2011 to 2,242 2015 [12]. While a European Food Safety Authority report concluded that fish (10.4\%), meat $(2.1 \%)$ and cheese $(0.5 \%)$ as foods that had the highest frequency of contamination of RTE from the whole RTE foods sampled [13].

Most importantly, listeriosis is a relatively rare illness compared to other foodborne infections; it is under-reported because surveillance activities are lacking [14]. According to available prevalence studies, approximately $5.1 \%$ of dairy and RTE food samples were contaminated with L. monocytogenes [14]. Cases of listeriosis caused by consumption of RTE foods have been in existence for years in Africa, as indicated in earlier studies [14], the 2019 outbreak in South Africa and a meta-analysis article conducted in Africa on foodborne pathogens $[15,16]$. In addition, food contamination has been evidenced by literature is known to be one of the principal vehicles of $L$. monocytogenes infections, especially in RTE foods, because of the absence of a heating step before consumption [17].

L. monocytogenes is resistant to some commercially available antibiotics and sanitizers [18]. Antibiotic resistance is increasingly becoming diverse [19] due to increased antibiotic usage [20]. Resistance rates in some European countries exceed 40-50\%; moreover, antibiotic resistance also contributes to the unsuccessful treatment of infections, leading to more severe and prolonged illnesses [21]. Furthermore, this causes production reduction, which affects livelihoods, including food security. In addition, the increase in global trade and travel necessitates the spread of antimicrobial resistance between countries and continents. The United Nations, in September 2016, indicated a global rise in antimicrobial resistance as a threat to health and human development [21].

Other authors have done meta-analysis and systematic reviews on this subject; a gap still exists regarding the estimates of the prevalence of $L$. monocytogenes in RTE foods and their antibiotic resistance profile in RTE foods $[22,23]$. According to the WHO, listeriosis is one of the most important zoonotic diseases globally $[7,15,24]$. Therefore, knowing the antibiotic-resistant $L$. monocytogenes in various RTE foods is cardinal in treating listeriosis in humans [25]. Furthermore, this may help guide policy on prudent use of antibiotics, especially those administered both in humans and animals [26].

This meta-analysis aimed to bring out the global perspective of antibiotic-resistant $L$. monocytogenes prevalence in assorted RTE foods by synthesizing published data. It also aimed to identify the RTE foods that are more likely to be contaminated with L. monocytogenes and the antibiotic resistance profiles in the different RTE foods.

\section{Materials and Methods}

\section{Ethical approval}

This is a meta-analysis study, and it does not require ethical approval.

\section{Study period and location}

This systematic/desk review was done in Zambia, Lusaka; however, the literature search on published data was based on citations from studies conducted from January 2010 to October 2020. The data analysis was conducted using Stata Version 15 (Stata Corporation, TX, USA).

\section{Search strategy}

We used Cochrane protocols to conduct a meta-analysis [27]. According to the PRISMA protocols, we selected and extracted them (Figure-1) $[28,29]$. Searching literature involved restricting peer-reviewed published articles in PubMed, Science Direct, Google Scholar, and Scopus on papers reporting $L$. monocytogenes in RTE foods and antibiotic-resistant profiles from January 1, 2010, to October 2020. Keywords such as: "prevalence" or "occurrence," or "Bacteria," "Listeria," "L. monocytogenes in RTE foods," and "antibiotic resistance in RTE foods" were used to search the databases. The extracted articles formed a reference list that the authors used for further screening to obtain additional relevant publications.

\section{Inclusion/exclusion criteria}

Criteria used for the inclusion of the article: 1 . English -language full text; 2. cross-sectional and descriptive studies; 3 . articles with known sample size; and studies reported the prevalence of either RTE foods or antibiotic resistance or reported both; 4 studies reported both RTE foods and raw foods and five studies that reported other bacteria, including 
L. monocytogenes. The authors excluded all books, workshop, and thesis due to a lack of peer review [30].

\section{Data extraction}

Data extracted from all considered articles included: the year of study, author name, country, data of the study, name of RTE foods, name of the antibiotic, total sample size, positive sample size, sample source, sampling technique, and method of isolation.

\section{Meta-analysis data}

Data were entered first in the Microsoft Excel spreadsheet for collation and cleaning and later exported into Stata software (Stata Corporation) for data analysis. Studies found were summarized using counts in a flow chart, and then cross-tabulations between food types and antibiotic resistance were performed to check for patterns. Meta-analysis was performed on all eligible studies to establish the global pooled prevalence of $L$. monocytogenes contamination of RTE foods and the associated antibiotic resistance profiles. The stratification of the meta-analysis was according to the type of RTE food, the country where the study originated, and the types of antibiotics used. Food grouping active types, such as fish, beef, chicken, and dairy RTE products, reduces the differences that may arise due to heterogeneity in the meta-analysis. We used the $\mathrm{I}^{2}$ to determine the heterogeneity of the studies, and if the $\mathrm{I}^{2}$ was $>50 \%$, heterogeneity was considered [31].

\section{Results}

\section{Literature search}

As depicted in Figure-1, a total of 2636 articles were identified from four peer-reviewed electronic databases: PubMed (388), Science Direct (43), Google Scholar (2162), and Scopus (38) published electronically dated back from January 2010 to October 2020. These 1236 duplicates identified were removed using EndNote $9 \times$ referencing software (Philadelphia, PA, USA). Thus, a total number of 164 articles remained after excluding redundant articles, of which 18 more were excluded due to lack of relevance of their title, not falling under any stipulated inclusion criteria stated by the authors. In addition, we further revised the full text of the 146 remaining articles. Furthermore, we further excluded 114 articles due to a lack of essential data, such as positive sample size or total sample size. Finally, only 32 articles reported the prevalence of L. monocytogenes and 11 articles reported on both the prevalence of $L$. monocytogenes and antibiotic profiles of $L$. monocytogenes considered appropriate for this meta-analysis, making the total number of studies to be 43 .

\section{Distribution of the type of RTE foods across studies}

Table-1 shows the number of publications from the continents that we included in this meta-analysis. Europe and Asia had more papers ( $\mathrm{n}=15$ and 12,

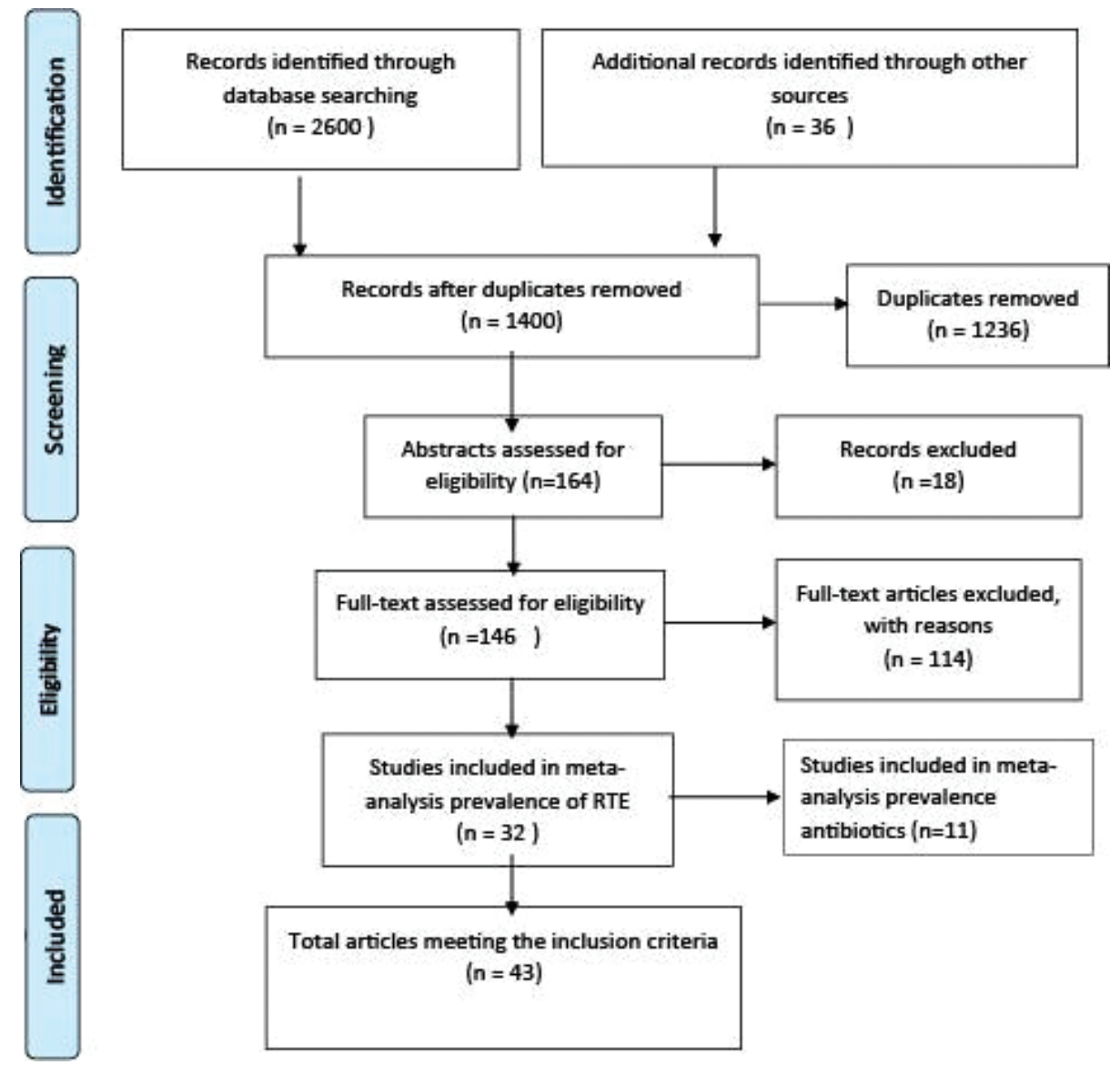

Figure-1: The flow diagram of the literature search and selection of eligible studies. 
respectively) than other continents, while Africa had 7, with North and South America reporting two papers each.

The food products differed in terms of their origin, type, and risk of L. monocytogenes contamination. Subsequently, we grouped these food items into a broader range of commodities for analysis. Most of the studies in Europe reported on Fish products, RTE salads, beef products and assorted RTE foods, while in Asia, the highest reported RTE foods were chicken products, beef products, assorted RTE foods, and fish products. While in Africa, the food with the highest frequency of reporting was chicken products and milk products, while North and South America had vegetable products and salads (Table-1).

\section{Distribution of antibiotics resistance across different types of RTE foods}

We studied various antibiotics for resistance in different RTE foods, and antibiotics we grouped into different classes that were homogeneous. The total number of antibiotics reported after grouping was 13 , while the number of food types was 4 . The antibiotics reported with many papers were penicillins, quinolone, aminoglycosides, lincomycins, and macrolides. The foods reported exhibiting resistance to antibiotics were assorted RTE foods and fish products (Table-2).
Country prevalence of $L$. monocytogenes contamination RTE foods and global pooled prevalence

The global pooled prevalence contamination of RTE foods with L. monocytogenes was $10.8 \%$ (95\% CI 9.4-12.2). The prevalence of L. monocytogenes contamination in RTE varied by country, from $4.5 \%$ in Estonia Eastern Europe to $56.3 \%$ in Jordan Middle Eastern Asia. The heterogeneity across the studies was high at 71.1. However, the within-country heterogeneity ranged from homogeneous 0.0 in Italy and other countries to very high at 91.9 in Sweden. Table-3 summarizes prevalence, weights, and heterogeneity in studies by country and global pooled prevalence.

\section{The global pooled prevalence of antibiotic resistance} of $\boldsymbol{L}$. monocytogenes in RTE foods

The global pooled prevalence of antibiotic-resistant $L$. monocytogenes in RTE foods was $38.1 \%(95 \%$ CI 36.1-39.7). The prevalence ranged from $9 \%$ in Taiwan to $94.1 \%$ in South Korea. The heterogeneity in the studies was very high at 89.9 . Table- 4 summarizes the prevalence of antibiotic resistance in L. monocytogenes in RTE foods.

\section{Prevalence of $L$. monocytogenes contamination in RTE foods}

In terms of specific food products, L. monocytogenes contamination ranking was as follows: The highest was in chicken products at $22 \%(95 \% \mathrm{CI}$ $0-28)$, followed by milk products at $18 \%$ (95\% CI

Table-1: Number of publications reviewed by continent and the type of foods reported.

\begin{tabular}{|c|c|c|c|c|c|c|}
\hline \multirow[t]{2}{*}{ Food Type } & \multicolumn{6}{|c|}{ Number of publications continents } \\
\hline & $\begin{array}{l}\text { Europe } \\
(n=15)\end{array}$ & $\begin{array}{c}\text { Asia } \\
(n=12)\end{array}$ & $\begin{array}{l}\text { Africa } \\
(n=7)\end{array}$ & $\begin{array}{l}\text { North America } \\
\qquad(n=2)\end{array}$ & $\begin{array}{l}\text { South America } \\
\qquad(n=2)\end{array}$ & Total \\
\hline Beef products & 8 & 8 & - & & - & 16 \\
\hline Milk & 7 & 2 & 5 & - & - & 14 \\
\hline Fish & 14 & 8 & 2 & - & - & 24 \\
\hline Salads & 9 & 4 & 2 & - & 2 & 17 \\
\hline Pork & 4 & - & - & - & - & 4 \\
\hline Vegetable product & 2 & 4 & 1 & 4 & - & 11 \\
\hline Chicken product & 1 & 9 & 5 & - & - & 15 \\
\hline Assorted ready-to-eat foods & 7 & 8 & 3 & 2 & - & 20 \\
\hline Total & 52 & 43 & 18 & 6 & 2 & 121 \\
\hline
\end{tabular}

Table-2: Distribution of antibiotics resistance across different types of ready-to-eat foods.

\begin{tabular}{|c|c|c|c|c|c|}
\hline \multirow[t]{2}{*}{ Antibiotics } & \multicolumn{5}{|c|}{ Type of food } \\
\hline & Assorted foods & Poultry products & Ready-to-eat salads & Fish product & Total \\
\hline Aminoglycosides & 12 & 2 & 5 & 0 & 19 \\
\hline Antimycobacterials & 4 & 1 & 1 & 0 & 6 \\
\hline Carbapenem & - & 0 & 0 & 1 & 1 \\
\hline Cephalosporin's & 10 & 0 & 0 & 2 & 12 \\
\hline Fluoroquinolones & 1 & 0 & 1 & 0 & 2 \\
\hline Glycopeptide & 7 & 1 & 0 & 1 & 9 \\
\hline Lincomycin & 3 & 0 & 1 & 2 & 6 \\
\hline Macrolides & 6 & 1 & 2 & 1 & 10 \\
\hline Nitrofurantoin & 1 & 0 & 0 & 0 & 1 \\
\hline Oxytetracycline & 1 & 0 & 0 & 0 & 1 \\
\hline Penicillins & 20 & 3 & 2 & 7 & 32 \\
\hline Quinolone & 12 & 2 & 2 & 4 & 20 \\
\hline Sulfonamides & 5 & 1 & 0 & 1 & 7 \\
\hline Tetracycline & 9 & 1 & 2 & 3 & 15 \\
\hline Total & 91 & 12 & 16 & 22 & 141 \\
\hline
\end{tabular}


9-27) and the smallest amount of contamination was found in fish and pork products at $12 \%$ (95\% CI 9-15) and $8 \%$ (95\% CI 12-20), respectively. Table-5 summarizes the prevalence of $L$. monocytogenes contamination in specific RTE foods.

\section{The global pooled prevalence of contamination of RTE} foods with antibiotic-resistant $\boldsymbol{L}$. monocytogenes

In terms of $L$. monocytogenes contamination of RTE foods with antibiotic resistance, the highest resistance was from penicillins at $80 \%$ (95\% CI 57-64), followed by cephalosporin's at $47 \%$ (95\% CI $44-56 \%)$ and the lowest antibiotic resistance was for sulfonamides and carbapenem at $13 \%(95 \%$ CI $3-23) 6 \%$ (95\% CI 0-45), respectively. Table-6 summarizes the prevalence of antibiotic-resistant L. monocytogenes contamination in RTE foods.
Methods for isolating $L$. monocytogenes and antibiotic resistance

Most studies reviewed in this meta-analysis reported the use of internationally recognized isolation protocols recommended for the isolation of $L$. monocytogenes, International Standard Organization $(70 \%)$, culture and biochemical/polymerase chain reaction (PCR) (14.1\%), two-step enrichment (12.6\%), and conventional methods (3.4\%).

Although antibiotic resistance was determined using several methods, the majority utilized disk diffusion on Mueller-Hinton agar (39\%), PCR (30\%), Kirby-Bauer method $(21.5 \%)$, pulsed-field gel electrophoresis method (4.7\%), and the disk diffusion method, which followed the Clinical and Laboratory Standards Institute with no specifications of agar used

Table-3: Country prevalence of Listeria monocytogenes contamination ready-to-eat foods and global pooled prevalence.

\begin{tabular}{|c|c|c|c|c|c|c|c|c|c|}
\hline Country & $\begin{array}{l}\text { No. of } \\
\text { studies }\end{array}$ & $\mathbf{E S}^{\mathbf{a}}$ & Lower & Upper & Weight & Heterogeneity & $\begin{array}{l}\text { Degrees of } \\
\text { freedom }\end{array}$ & p-value & $\mathbf{I}^{2}(\%) \mathrm{d}$ \\
\hline Spain & 3 & 0.090 & 0.045 & 0.131 & 11.52 & 19.19 & 7 & 0.008 & 63.5 \\
\hline Italy & 2 & 0.271 & 0.194 & 0.347 & 3.31 & 3.28 & 4 & 0.512 & 0.0 \\
\hline Japan & 2 & 0.063 & 0.004 & 0.121 & 5.76 & 0.84 & 10 & 1.000 & 0.0 \\
\hline Turkey & 2 & 0.110 & -0.045 & 0.268 & 0.78 & 0.10 & 3 & 0.992 & 0.0 \\
\hline Sebia & 1 & 0.251 & 0.146 & 0.355 & 1.78 & 1.72 & 1 & 0.190 & 41.7 \\
\hline Northern Ireland & 1 & 0.084 & -0.010 & 0.178 & 2.20 & 0.82 & 2 & 0.664 & 0.0 \\
\hline Sweden & 1 & 0.232 & 0.175 & 0.289 & 6.01 & 49.22 & 4 & 0.000 & 91.9 \\
\hline Greece & 2 & 0.081 & -0.055 & 0.217 & 1.05 & 0.46 & 4 & 0.977 & 0.0 \\
\hline Estonia & 1 & 0.045 & 0.022 & 0.67 & 38.96 & 44.82 & 7 & 0.000 & 84.4 \\
\hline Iran & 3 & 0.276 & 0.186 & 0.365 & 2.44 & 48.56 & 5 & 0.000 & 89.7 \\
\hline Egypt & 1 & 0.233 & -0.010 & 0.476 & 0.33 & 0.64 & 1 & 0.423 & 0.0 \\
\hline India & 1 & 0.052 & -0.225 & 0.329 & 0.25 & 0.02 & 1 & 0.886 & 0.0 \\
\hline Algeria & 1 & 0.063 & -0.114 & 0.240 & 0.62 & 0.12 & 1 & 0.732 & 0.0 \\
\hline Ethiopia & 1 & 0.089 & -0.059 & 0.237 & 0.89 & 0.24 & 3 & 0.971 & 0.0 \\
\hline United Kingdom & 3 & 0.133 & 0.075 & 0.191 & 5.85 & 11.58 & 5 & 0.041 & 56.8 \\
\hline Malaysia & 5 & 0.139 & 0.079 & 0.199 & 5.48 & 12.64 & 18 & 0.812 & 0.0 \\
\hline Brazil & 2 & 0.176 & 0.0 & 0.049 & 0.304 & 1.20 & 21.61 & 0.001 & 76.9 \\
\hline Nigeria & 2 & 0.241 & 0.159 & 0.322 & 2.97 & 23.66 & 3 & 0.000 & 87.3 \\
\hline China & 5 & 0.053 & -0.012 & 0.118 & 4.58 & 1.31 & 6 & 0.971 & 0.0 \\
\hline Thailand & 1 & 0.147 & 0.022 & 0.273 & 1.24 & 0.33 & 1 & 0.563 & 0.0 \\
\hline Jordon & 1 & 0.563 & 0.426 & 0.699 & 1.06 & 14.82 & 2 & 0.001 & 86.5 \\
\hline Sudan & 2 & 0.124 & 0.018 & 0.230 & 1.74 & 1.20 & 4 & 0.879 & 0.0 \\
\hline Overall & 43 & 0.108 & 0.094 & 0.122 & 100.0 & 407.82 & 118 & $<0.000$ & 71.1 \\
\hline
\end{tabular}

Table-4: The global pooled prevalence of antibiotic resistance of Listeria monocytogenes in ready-to-eat foods.

\begin{tabular}{lccccccccc}
\hline Country & $\begin{array}{c}\text { No. of } \\
\text { studies }\end{array}$ & ES & Lower & Upper & $\begin{array}{c}\text { Weight of } \\
\text { ES (\%) }\end{array}$ & $\begin{array}{c}\text { Heterogeneity } \\
\text { statistic }\end{array}$ & $\begin{array}{c}\text { Degrees of } \\
\text { freedom }\end{array}$ & p-value & $\left.\mathbf{I}^{2}(\%)\right)^{\mathbf{d}}$ \\
\hline Brazil & 1 & 0.250 & 0.005 & 0.495 & 0.42 & 0.00 & 1 & 1.000 & 0.0 \\
China & 5 & 0.146 & 0.115 & 0.178 & 26.24 & 201.7 & 37 & 0.000 & 81.7 \\
Ethiopia & 1 & 0.487 & 0.344 & 0.631 & 1.24 & 5.80 & 3 & 0.122 & 48.3 \\
Iran & 2 & 0.267 & 0.225 & 0.308 & 14.78 & 53.57 & 18 & 0.000 & 66.4 \\
Jordan & 1 & 0.121 & -0.137 & 0.378 & 0.38 & 0.13 & 2 & 0.936 & 0.0 \\
Korea & 1 & 0.941 & 0.883 & 1.00 & 7.44 & 24.52 & 1 & 0.000 & 95.9 \\
Malaysia & 1 & 0.802 & 0.696 & 0.908 & 2.27 & 7.32 & 2 & 0.026 & 72.7 \\
Nigeria & 2 & 0.469 & 0.441 & 0.496 & 33.62 & 120.67 & 19 & 0.000 & 84.3 \\
Spain & 1 & 0.164 & 0.004 & 0.324 & 0.99 & 0.59 & 6 & 0.997 & 0.0 \\
Taiwan & 1 & 0.091 & -0.306 & 0.488 & 0.16 & 0.00 & 0 & - & - \\
Turkey & 4 & 0.448 & 0.392 & 0.504 & 8.04 & 142.60 & 24 & 0.000 & 83.2 \\
Overall & 20 & 0.381 & 0.365 & 0.397 & 100.0 & 1342.08 & 136 & 0.000 & 89.9 \\
\hline
\end{tabular}

Effect size (ES): Prevalence of antibiotic resistance in ready-to-eat foods (ratio of positive samples/total samples).

The weight of ES is related to the total sample size of individual studies. Variation in study outcomes between studies. Heterogeneity across studies $\left(\mathrm{I}^{2}\right)$ 
Table-5: Prevalence of Listeria monocytogenes contamination in ready-to-eat foods.

\begin{tabular}{|c|c|c|c|c|c|c|c|}
\hline Item & Country & No. of Papers & Effect size & $95 \% \mathrm{CI}$ & Weight & I Squared (\%) & p-value \\
\hline & Spain & 2 & 0.25 & $0.08,0.43$ & 27.61 & 86.6 & $<0.001$ \\
\hline \multirow[t]{6}{*}{ Milk products } & Italy & 1 & 0.02 & $0.52,0.56$ & 2.75 & 0 & $<0.001$ \\
\hline & Sweden & 1 & 0.36 & $0.29,1.05$ & 1.79 & 0 & $<0.001$ \\
\hline & Estonia & 2 & 0.21 & $0.07,0.35$ & 41.28 & 0 & 0.578 \\
\hline & India & 2 & 0.05 & $0.23,0.33$ & 10.6 & 0 & 0.886 \\
\hline & Ethiopia & 2 & 0.07 & $0.15,0.30$ & 15.97 & 0 & 0.678 \\
\hline & Overall & 10 & 0.18 & $0.09,0.27$ & 100 & 46.5 & 0.044 \\
\hline \multirow[t]{12}{*}{ Fish products } & Spain & 2 & 0.08 & $0.02,0.14$ & 28.24 & 0 & 0.685 \\
\hline & Italy & 1 & 0.34 & $0.20,0.48$ & 5.66 & 0 & 0.001 \\
\hline & Japan & 1 & 0.08 & $-0.01,0.14$ & 21.13 & 0 & 0.999 \\
\hline & Turkey & 1 & 0.12 & $-0.25,0.49$ & 0.8 & 0 & 0.001 \\
\hline & Sebia & 1 & 0.25 & $0.15,0.36$ & 9.88 & 41.7 & 0.19 \\
\hline & Northern Ireland & 1 & 0.01 & $-0.31,0.34$ & 0.01 & 0 & 0.001 \\
\hline & Sweden & 1 & 0.12 & $0.06,0.19$ & 25.84 & 0 & 0.449 \\
\hline & Greece & 1 & 3.34 & $-0.12,0.24$ & 3.34 & 0 & 0.923 \\
\hline & Estonia & 1 & 0.06 & $-0.42,0.53$ & 0.48 & 0 & 0 \\
\hline & Iran & 1 & 0.06 & $-0.20,0.30$ & 1.78 & 12.4 & 0 \\
\hline & Egypt & 1 & 0.23 & $-0.01,0.48$ & 1.84 & 0.423 & 0 \\
\hline & Overall & 12 & 0.12 & $0.09,0.15$ & 100.0 & 12.4 & 0.289 \\
\hline Pork products & Spain & 1 & 0.08 & $0.03,0.14$ & 100 & 0 & 0.736 \\
\hline \multirow{10}{*}{ Ready-to-eat salads } & Italy & 1 & 0.27 & $0.16,0.38$ & 13.91 & 0 & 0 \\
\hline & Turkey & 1 & 0.18 & $-0.35,0.71$ & 0.56 & 0 & 0 \\
\hline & Northern Ireland & 1 & 0.04 & $-0.13,0.20$ & 5.8 & 0 & 0 \\
\hline & Estonia & 1 & 0.19 & $0.11,0.26$ & 27.69 & 0 & 0 \\
\hline & Iran & 1 & 0.31 & $0.03,0.59$ & 1.96 & 0 & 0 \\
\hline & United Kingdom & 1 & 0.04 & $-0.06,0.13$ & 17.13 & 0 & 0 \\
\hline & Malaysia & 3 & 0.14 & $0.07,0.22$ & 26.31 & 25.4 & 0.235 \\
\hline & Brazil & 2 & 0.68 & $0.38,098$ & 1.76 & 85 & 0.001 \\
\hline & Nigeria & 2 & 0.2 & $0.02,0.38$ & 4.89 & 46.6 & 0.171 \\
\hline & Overall & 13 & 0.16 & $0.12,0.20$ & 100.0 & 62.4 & $<0.001$ \\
\hline \multirow[t]{10}{*}{ Beef products } & Japan & 1 & 0.07 & $0.21,0.35$ & 0.58 & 0 & 0.787 \\
\hline & Northern Ireland & 1 & 0.12 & $-0.00,0.24$ & 3.08 & 0 & 0 \\
\hline & Sweden & 1 & 0.61 & $0.49,0.73$ & 3.11 & 0 & 0 \\
\hline & Estonia & 1 & 0.02 & $-0.00,0.04$ & 80.32 & 0 & 0 \\
\hline & Iran & 2 & 0.33 & $0.21,0.45$ & 3.13 & 95.5 & 0.001 \\
\hline & United Kingdom & 1 & 0.02 & $-0.16,0.20$ & 1.4 & 0.991 & 0.001 \\
\hline & Malaysia & 1 & 0.07 & $-0.28,0.41$ & 0.39 & 0 & 0 \\
\hline & China & 4 & 0.05 & $0.03,0.12$ & 7.75 & 0 & 0.743 \\
\hline & Thailand & 1 & 0.27 & $-0.16,0.71$ & 0.24 & 90.3 & 0.001 \\
\hline & Overall & 13 & 0.16 & $0.12,0.20$ & 100.0 & 62.4 & $<0.001$ \\
\hline \multirow[t]{7}{*}{ Chicken products } & Turkey & 1 & 0.1 & $-0.11,0.32$ & 7.7 & 0 & 0 \\
\hline & Iran & 1 & 0.26 & $0.07,0.45$ & 9.93 & 0 & 0 \\
\hline & Malaysia & 1 & 0.22 & $0.05,0.40$ & 11.64 & 0 & 0.972 \\
\hline & China & 2 & 0.07 & $-0.06,0.20$ & 20.5 & 0 & 0.965 \\
\hline & Jordan & 1 & 0.56 & $-0.43,0.70$ & 18.95 & 86.5 & 0.001 \\
\hline & Sudan & 1 & 0.12 & $-0.02,0.23$ & 31.28 & 0 & 0.879 \\
\hline & Overall & 7 & 0.22 & $-0.16,0.28$ & 100.0 & 72.1 & $<0.001$ \\
\hline \multirow[t]{12}{*}{ Assorted ready-to-eat } & Italy & 1 & 0.1 & $-0.19,0.39$ & 2.44 & 0 & 0 \\
\hline & Japan & 1 & 0.09 & $-0.05,0.24$ & 9.85 & 0 & 0 \\
\hline & Greece & 1 & 0.1 & $-0.10,0.31$ & 4.86 & 0 & 0.986 \\
\hline & Estonia & 1 & 0.47 & $0.26,0.67$ & 5.09 & 80.7 & 0.023 \\
\hline & Algeria & 1 & 0.14 & $-0.34,0.83$ & 0.9 & 0 & 0 \\
\hline & Ethiopia & 1 & 0.1 & $0.10,0.30$ & 5.45 & 0 & 0.916 \\
\hline & United Kingdom & 3 & 0.22 & $0.14,0.30$ & 33.65 & 0 & 0.411 \\
\hline & Malaysia & 1 & 0.1 & $-0.04,0.23$ & 11.64 & 0 & 0.609 \\
\hline & Nigeria & 1 & 0.48 & $0.35,0.61$ & 12.06 & 0 & 0 \\
\hline & China & 1 & 0.06 & $-0.28,0.41$ & 1.8 & 0 & 0 \\
\hline & Thailand & 1 & 0.14 & $0.01,0.27$ & 12.24 & 54.7 & 0.002 \\
\hline & Overall & 13 & 0.21 & $0.16,0.25$ & 100.0 & 54.7 & 0.002 \\
\hline
\end{tabular}

(3.4\%) and the least was the micro-broth dilution method (2\%).

\section{Discussion}

We synthesized the prevalence of L. monocytogenes in RTE food and antibiotic-resistant profiles globally using peer-reviewed published data in this systematic review and meta-analysis. Based on the meta-analysis results, the overall global pooled estimate of $L$. monocytogenes prevalence in RTE foods was $10.8 \%$. In comparison, the global pooled estimate of antibiotic resistance in RTE was $38.1 \%$ among 
Table-6: The global pooled prevalence of contamination of ready-to-eat foods with antibiotic-resistant Listeria monocytogenes.

\begin{tabular}{|c|c|c|c|c|c|c|c|}
\hline Item & Country & No. of papers & Effect size & $95 \% \mathrm{CI}$ & Weight & I Squared (\%) & p-value \\
\hline \multirow[t]{8}{*}{ Aminoglycosides } & China & 7 & 0.07 & $-0.00,0.14$ & 60.75 & 0.0 & 0.985 \\
\hline & Iran & 2 & 0.10 & $-0.17,0.28$ & 1.77 & 0.0 & 0.855 \\
\hline & Italy & 2 & 0.06 & $0.17,0.28$ & 6.27 & 0.0 & 0.855 \\
\hline & Jordan & 1 & 0.06 & $-0.41,0.52$ & 1.46 & 0.0 & $<0.001$ \\
\hline & Nigeria & 2 & 0.26 & $0.11,0.40$ & 14.56 & 39.3 & 0.176 \\
\hline & Spain & 1 & 0.21 & $0.25,0.68$ & 1.48 & 0.0 & $<0.001$ \\
\hline & Turkey & 2 & 0.39 & $0.24,0.55$ & 13.70 & 83.0 & 0.001 \\
\hline & Overall & 17 & 0.07 & $-0.00,0.14$ & 60.75 & 0.0 & 0.985 \\
\hline \multirow[t]{5}{*}{ Antimycobacterials } & China & 3 & 0.05 & $-0.06,0.15$ & 55.38 & 0.0 & 0.832 \\
\hline & Iran & 1 & 0.08 & $-0.11,0.27$ & 17.84 & 0.0 & $<0.001$ \\
\hline & Malaysia & 1 & 0.83 & $0.66,1.00$ & 21.40 & 0.0 & $<0.001$ \\
\hline & Turkey & 1 & 0.47 & $0.12,0.82$ & 5.38 & 0.0 & $<0.001$ \\
\hline & Overall & 6 & 0.24 & $0.16,0.32$ & 100.0 & 91.9 & 0.001 \\
\hline Carbapenem & Turkey & 1 & 0.06 & $-0.37,0.49$ & 100.0 & 0.0 & $<0.001$ \\
\hline \multirow[t]{6}{*}{ Cephalosporin's } & China & 3 & 0.52 & $0.44,0.59$ & 14.61 & 95.9 & 0.001 \\
\hline & Italy & 1 & 0.15 & $-0.36,0.44$ & 0.52 & 0.0 & $<0.001$ \\
\hline & Nigeria & 1 & 0.47 & $0.44,0.50$ & 82.99 & 0.0 & 0.990 \\
\hline & Spain & 1 & 0.21 & $-0.25,0.67$ & 0.39 & 0.0 & $<0.001$ \\
\hline & Turkey & 1 & 0.15 & $-0.08,0.39$ & 1.49 & 0.0 & 0.678 \\
\hline & Overall & 7 & 0.47 & $0.44,0.50$ & 100.0 & 88.3 & 0.001 \\
\hline Fluoroquinolones & Iran & 2 & 0.23 & $0.11,0.35$ & 100.0 & 0.0 & 0.430 \\
\hline \multirow[t]{7}{*}{ Glycopeptide } & China & 2 & 0.02 & $-0.13,0.16$ & 33.37 & 0.0 & 0.925 \\
\hline & Iran & 1 & 0.35 & $0.19,0.51$ & 27.68 & 0.0 & $<0.001$ \\
\hline & Italy & 1 & 0.06 & $-0.15,0.27$ & 15.36 & 0.0 & 0.908 \\
\hline & Nigeria & 1 & 0.57 & $0.23,0.91$ & 5.87 & 0.0 & $<0.001$ \\
\hline & Spain & 1 & 0.21 & $-0.25,0.67$ & 3.25 & 0.0 & $<0.001$ \\
\hline & Turkey & 2 & 0.49 & $0.27,0.71$ & 14.46 & 67.1 & 0.081 \\
\hline & Overall & 8 & 0.22 & $0.14,0.31$ & 100.0 & 68.2 & 0.001 \\
\hline \multirow[t]{6}{*}{ Lincomycins } & China & 1 & 0.34 & $0.16,0.52$ & 28.49 & 0.0 & $<0.001)$ \\
\hline & Iran & 2 & 0.06 & $-0.07,0.20$ & 49.77 & 0.0 & 0.761 \\
\hline & Italy & 1 & 0.28 & $0.02,0.53$ & 13.53 & 0.0 & $<0.001$ \\
\hline & Korea & 1 & 0.28 & $0.02,0.53$ & 0.0 & 0.0 & $<0.001$ \\
\hline & Turkey & 2 & 0.13 & $-0.20,0.47$ & 8.21 & 0.0 & $<0.001$ \\
\hline & Overall & 7 & 0.17 & $0.08,0.27$ & 100.0 & 39.9 & 0.001 \\
\hline \multirow[t]{11}{*}{ Penicillins } & China & 3 & 0.05 & $-0.03,0.12$ & 17.78 & -0.0 & 0.981 \\
\hline & Ethiopia & 1 & 0.87 & $0.44,0.90$ & 2.07 & 0.0 & $<0.001$ \\
\hline & Iran & 2 & 0.30 & $0.31,0.48$ & 15.12 & -0.0 & 0.435 \\
\hline & Italy & 1 & 0.47 & $0.36,0.50$ & 8.60 & -88.8 & 0.001 \\
\hline & Korea & 1 & 0.97 & $0.911,1.3$ & 31.08 & 0.0 & $<0.001$ \\
\hline & Malaysia & 1 & 0.97 & $0.91,1,03$ & 0.0 & 0.0 & $<0.001$ \\
\hline & Nigeria & 2 & 0.88 & $0.78,0.90$ & 10.45 & -0.0 & 0.58 \\
\hline & Spain & 1 & 0.07 & $-0.25,0.37$ & 1.24 & 0.0 & $<0.001$ \\
\hline & Taiwan & 1 & 0.09 & $-0.31,0.49$ & 0.70 & 0.0 & $<0.001$ \\
\hline & Turkey & 3 & 0.89 & $0.50,0.78$ & 11.58 & -93.5 & $<0.001$ \\
\hline & Overall & 16 & 0.80 & $0.57,0.64$ & 100.0 & 94.9 & 0.001 \\
\hline \multirow[t]{7}{*}{ Quinolones } & China & 3 & 0.09 & $-0.01,0.18$ & 33.37 & 0.0 & 0.771 \\
\hline & Ethiopia & 1 & 0.37 & $0.15,0.60$ & 5.74 & 50.2 & 0.156 \\
\hline & Iran & 2 & 0.18 & $0.08,0.29$ & 27.00 & 41.3 & 0.182 \\
\hline & Nigeria & 2 & 0.20 & $0.08,0.31$ & 21.46 & 29.6 & 0.234 \\
\hline & Spain & 1 & 0.21 & $-0.25,0.68$ & 1.35 & 0.0 & $<0.001$ \\
\hline & Turkey & 3 & 0.41 & $0.25,0.57$ & 11.07 & 49.5 & 0.008 \\
\hline & Overall & 12 & 0.19 & $0.14,0.24$ & 100.0 & 49.5 & 0.008 \\
\hline \multirow[t]{6}{*}{ Sulfonamides } & China & 2 & 0.04 & $-0.08,0.15$ & 73.00 & 0.0 & 0.976 \\
\hline & Malaysia & 1 & 0.39 & $0.07,0.71)$ & 9.39 & 0.0 & $<0.001$ \\
\hline & Nigeria & 1 & 0.57 & $-0.23,0.91$ & 8.09 & 0.0 & $<0.001$ \\
\hline & Spain & 1 & 0.21 & $-0.25,0.68$ & 4.42 & 0.0 & $<0.001$ \\
\hline & Turkey & 1 & 0.81 & $-0.25,0.61$ & 5.10 & 0.0 & $<0.001$ \\
\hline & Overall & 6 & 0.13 & $0.03,0.23$ & 100.0 & 48.5 & 0.070 \\
\hline \multirow[t]{6}{*}{ Tetracycline's } & China & 4 & 0.09 & $-0.02,0.19$ & 29.93 & 0.0 & 0.810 \\
\hline & Ethiopia & 1 & 0.38 & $0.06,0.69$ & 3.20 & 0.0 & $<0.001$ \\
\hline & Iran & 2 & 0.22 & $0.10,0.34$ & 21.17 & 82.7 & 0.016 \\
\hline & Italy & 2 & 0.04 & $-0.19,0.26$ & 6.50 & 0.0 & 0.913 \\
\hline & Jordan & 1 & 0.12 & $-0.33,0.56$ & 1.60 & 0.0 & $<0.001$ \\
\hline & Korea & 1 & 0.18 & $-0.13,0.49$ & 1.60 & 0.0 & $<0.001$ \\
\hline
\end{tabular}


Table-6: (Continued).

\begin{tabular}{llcccccc}
\hline Item & Country & No. of papers & Effect size & $\mathbf{9 5 \%}$ CI & Weight & I Squared (\%) & p-value \\
\hline & Malaysia & 1 & 0.87 & $0.72,1.02$ & 14.80 & 0.0 & $<0.001$ \\
& Nigeria & 1 & 0.71 & $0.43,1.00$ & 4.05 & 0.0 & $<0.001$ \\
& Turkey & 2 & 0.30 & $0.14,0.46$ & 12.69 & 15.4 & 0.277 \\
& Overall & 15 & 0.30 & $0.24,0.35$ & 100.0 & 84.8 & 0.001 \\
\hline
\end{tabular}

all contaminated samples. Thus, we conducted such a review study on finding the global prevalence of L. monocytogenes and its antibiotic profiles in RTE foods for the $1^{\text {st }}$ time to the best of our knowledge. Further, this study sub-analyzed the global prevalence by type of food as well as regionally by continent. We believe this study has contributed to the body of knowledge on food safety and alerts policymakers in different continents on food contamination levels with L. monocytogenes in their regions and globally.

Comparatively, a meta-analysis conducted among Iranian studies revealed a much lower prevalence of $4 \%$ despite having the same number of included studies of 32 with this current review [32]. However, the variance in the pooled prevalence between the two reviews were attribute to the food types included; the review from Iran did not limit its foods to RTE foods only but also included raw foods such as milk and kinds of seafood with some assortment of RTE foods. In contrast, this current review only reported on various RTE foods. L. monocytogenes is a post-contaminating bacterium that has a high chance of soiling RTE foods because they undergo multiple processing stages, which are likely to increase their risk of contamination which may partly explain the discrepancy [33]. In addition, the Iranian review focused on studies done within Iran and the foods included may have some level of similarity in terms of environmental factors, which may to a greater extent, decrease the risk of L. monocytogenes contamination compared to this current review which had a wide range of studies that came from different environmental exposures. Luber's work cited that the differences in environmental factors equally influence the proportion of contamination in a given setting [34]. Primarily, $70 \%$ of the studies in this review reported using internationally accepted isolation, standards that have been reported to yield high discriminatory power compared to other methods, which may have contributed to the increased rate of positivity observed in the synthesized studies [35].

L. monocytogenes prevalence was highest in RTE chicken products (22\%) compared to the other RTE foods analyzed in this study. Study on chicken products reported to have utilized more than one enrichment broth to increase the sensitivity of the detection of L. monocytogenes to about $97.5-98.9 \%$ compared to the expected results of $60-70 \%$ when standard regulatory protocols are used [36]. Enrichment media used may inhibit the detection of L. monocytogenes because the growth rate is usually slow and below detectable levels depending on the type used [36]. Listeria, including L. monocytogenes, may exist at deficient levels in foods; therefore, sample enrichment protocols must amplify these initial populations to levels that are detectable [37]. RTE salads and beef products had the least pooled prevalence. These studies had an assortment of protocols used, and the majority did not mention the specific media used for isolation. On the other hand, some selective agents used in enrichment may inhibit the repair and detection of sublethally injured Listeria through processing treatments such as heating [36]. These differences in protocols may have influenced the isolation variances in other RTE foods in this review study.

Notably, the distribution of studies reported on the RTE chicken products showed some similarity in geographic settings, with more studies from Asia (5), Europe, and Africa (1). Asian countries, although others like China, are still regarded as developing countries that have considerably high technological equipment and machinery for the detection of fastidious bacteria such as $L$. monocytogenes coupled with increased surveillance of foodborne diseases. The advancement in technology may have influenced the positivity rate described above, with more contamination recorded in RTE chicken products in this current review. Most importantly, poultry products are among the most critical sources of Listeria spp. Especially L. monocytogenes [38]. Novelists from original studies done by Osaili et al. [34] reported a higher prevalence of $L$. monocytogenes of $33.3 \%$ in RTE poultry, while Wong et al. [35] reported from chicken burgers and luncheon product samples $20-13.3 \%$. Isolation prevalence in the studies described above with this review is inferred mainly due to differences in the processing methods used on the RTE chicken product $[39,40]$.

Another remarkable finding was the high heterogeneity among the studies that reported the Prevalence of RTE foods, ranging from 0.0 to about $91.9 \%$. The majority of the studies included in this review reported on different types of RTE foods and publication skewness within the countries. As previously indicated by other authors, the type of food influences the risk of L. monocytogenes contamination [34]. We may speculate the reasons for the high prevalence of L. monocytogenes in this review in some RTE foods such as chicken products, assorted RTE, and milk products to have been influenced by inadequate heat treatment, poor sanitation, inadequate physical separation between the raw and cooked food areas, and cross-contamination during processing and handling. 
In addition, $L$. monocytogenes contamination, as indicated by the results of a study done on milk products, is likely to be lower in milk than in food products such as meat and other meat products [41]. The studies in this review, despite coming from the same continent, a number of them had geographical distribution differences; for instance, the studies that came from Asia mainly came from the Eastern part.

In contrast, in Africa majority of the studies came from the Northern part with a slight difference from studies that came from Europe but with a range of some similarities. Sample size differences in the reviewed studies also played a role in contributing to the high heterogeneity observed. Adequate sample size estimation should be considered from the initial study plan in order to ensure its validity and power[42].

This review indicated that the highest resistance in various RTE foods came from penicillins (80\%) and the least in sulfonamides (13\%) (Table-2). Most of the antibiotics studied belonged to the class of penicillins, cephalosporins, and tetracycline. Cephalosporin and tetracycline are third-generation antibiotics intrinsically resistant to L. monocytogenes; these are considered inappropriate for treatment for listeriosis in infants and pregnant women [24]. Penicillin and other antibiotics such as ampicillin and oxacillin are the most well-known active $\beta$-lactams capable of inhibiting the synthesis of the bacterial cell wall of peptidoglycan [24]. L. monocytogenes naturally susceptible to $\beta$-lactams and the prescribed standard antibiotic therapy for treating listeriosis includes penicillin/ ampicillin combined or alone together with an aminoglycoside (gentamicin) [43]. In this current review, $L$. monocytogenes resistance was highest in $\beta$-lactam antibiotics, which is a public health concern in terms of human treatment of listeriosis.

Most of these named classes of antibiotics are susceptible to L. monocytogenes resistance. Furthermore, L. monocytogenes is naturally resistant to the currently used third and fourth generation fluoroquinolones and cephalosporin's [25]. Over the years, there has been increasing recognition of widespread antibiotic use in agriculture and aquaculture in most countries, including the ones included in this study [44]. Processing steps during food production such as slaughtering practices, farming activities and transportation of food animals may introduce resistant bacteria into the food chain [45]. Moreover, contamination during food preparation and consumption of contaminated meat and other food products may develop antibiotic-resistant bacteria in humans. Factors contributing to the rise in antibiotic-resistant bacterial species are complex and consist of various aspects. Some factors are unavoidable and inherent, such as the ability of the bacteria to adapt rapidly to changing environmental conditions because of their short generation time and the intrinsic resistance of certain bacteria. However, some variables are human, such as the extensive use of antibiotics as a growth promoter in farming practice. A significant concern to human health is the transfer of antibiotic-resistant bacteria present in food to humans and subsequent colonizing the gastrointestinal tract [45]. Overlap of use for other purposes of human medicine has also contributed to the development of resistance to antibiotics commonly [46,47]. The use of antibiotics in the food production chain is necessary, especially in export food countries [48].

Many antibiotics used in foods are the same surrogates as antibiotics used in human therapeutics; this partly explains why high antibiotic resistance in RTE foods in this review was recorded [49]. Stonsaovapak and Boonyaratanakornkit [45] reported ampicillin and rifampicin resistance in $L$. monocytogenes isolated from RTE foods. At the same time, in Columbia, a study was conducted on various RTE foods and resistance was recorded in clindamycin, streptomycin and amikacin [46]. The resistance of L. monocytogenes was evident in milk products to some commonly used antibiotics such as penicillin, ampicillin, tetracycline, and gentamicin, which agrees with the findings of this study $[47,48]$. L. monocytogenes are intrinsically resistant to certain antibiotics and reported by others confirming the same [1]. The picture given from the above reported prevalence from other studies is depicting a similar resistance pattern of the antibiotics with this current review study.

Globalization and exportation of foods from one country to another have also increased production using antibiotic growth promoters [26]. Most of these countries are among the highest exporters of most foods and meet the demand for food supply antibiotics. However, several foods are likely to contain antibiotic residues, especially where regulations are lacking [44]. Many countries, particularly the EU, have made substantial efforts to reduce the overall use of antibiotics in food-producing animals by benchmarking antibiotic use at the farm level and encouraging antibiotic stewardship. As a result, some foods, such as those coming from animal sources, are more prone to antibiotic use than others [21]. However, cautious use of antibiotics is needed to ensure we met regulations in food products [44]. The other underlying factor is country variations regarding the availability of guidelines on the criteria for purchasing certain drugs such as penicillin [50]. Meanwhile, most developed countries use antibiotics as growth promoters in food animals, including feed, to boost global production; this explains the country variations recorded in the current review [26].

High heterogeneity among the studies that reported antibiotic resistance was equally observed. The differences in antibiotics studied across the studies, sample size, and the sample's origin (country) can ascribe to the above observation [49]. Easy purchase of some antibiotics such as penicillin, compared to sulfonamides antibiotics, contributes to high levels of antibiotic resistance on some groups of antibiotics 
compared to others. Antibiotics prescribed widely are more prone to develop resistance with time, mainly when not used according to their prescription [44]. More importantly, there is a need for susceptibility testing before some high-priority antibiotics in food products are used to curb increased antibiotic resistance. As previously mentioned, L. monocytogenes are naturally resistant to most antibiotics used in human and animal foods [25].

\section{Conclusion}

This study revealed a considerably high pooled prevalence of $L$. monocytogenes in various RTE foods, and, more specifically, the RTE foods that had the highest pooled prevalence were chicken products. While the antibiotics with the highest pooled prevalence was penicillin. Therefore, control measures need to be put in place to reduce the risk of listeriosis due to consumption of RTE foods, especially chicken products. The findings also show that RTE foods are a vital source of antibiotic-resistant $L$. monocytogenes, which is a public health concern.

\section{Authors' Contributions}

PM: Conceived the design, analyzed the data, and revised the work. NM: Performed the cleaning of the dataset, carried out the statistical analysis, and reviewed the manuscript. MM: Contributed to a thorough review of the manuscript and supervision. ARM: Played a significant role in the early drafting and proofreading of the manuscript. WM: Involved in the proofreading of the manuscript. JBM: Contributed to manuscript drafting and proofreading. All authors read and approved the final manuscript.

\section{Acknowledgments}

The authors express their gratitude to the following partners: Higher Education Loans and Scholarships, Levy Mwanawasa Medical University, and the University of Zambia for the support rendered. In addition, the Africa Center of Excellence for Infectious Diseases of Humans and Animals (ACEIDHA) (Grant no. 5803-ZM) funded this research.

\section{Competing Interests} interests.

The authors declare that they have no competing

\section{Publisher's Note}

Veterinary World remains neutral with regard to jurisdictional claims in published institutional affiliation.

\section{References}

1. Rocourt, J. \& Buchrieser, C. (2007). The genus Listeria and Listeria monocytogenes: phylogenetic position, taxonomy, and identification. In Listeria, Listeriosis, and Food Safety, 3rd edn, pp. 1-20. Edited by E. T. Ryser \& E. H. Marth. Boca Raton, FL: CRC Press.

2. Roberts, B.N., Chakravarty, D., Gardner, J., Ricke, S.C. and
Donaldson, J.R. (2020) Listeria monocytogenes response to anaerobic environments. J. Pathog., 9(3): 210.

3. Sabil, R.M.S., Prahesti, K.I. and Yuliati, F.N. (2019) Behaviour of Listeria monocytogenes in pasteurization milk during refrigerator storage. Eur. J. Sustain. Dev., 8(4): 264-264.

4. Magalhães, R., Ferreira, V., Brandão, T.R., Palencia, R.C., Almeida, G. and Teixeira, P. (2016) Persistent and non-persistent strains of Listeria monocytogenes: A focus on growth kinetics under different temperature, salt, and $\mathrm{pH}$ conditions and their sensitivity to sanitizers. J. Food Microbiol., 57: 103-108.

5. Goulet, V., King, L.A., Vaillant, V. and de Valk, H. (2013) What is the incubation period for listeriosis? BMC Infect. Dis., 13: 11.

6. World Health Organization. (2015) WHO Estimates of the Global Burden of Foodborne Diseases: Foodborne Disease Burden Epidemiology Reference Group 2007-2015. World Health Organization, Geneva.

7. World Health Organization. (2016) Burden of Foodborne Diseases in the South-East Asia Region. World Health Organization, Geneva.

8. Tack, D.M., Ray, L., Griffin, P.M., Cieslak, P.R., Dunn, J., Rissman, T., Jervis, R., Lathrop, S., Muse, A., Duwell, M.J.M. and Report, M.W. (2020) Preliminary incidence and trends of infections with pathogens transmitted commonly through food foodborne diseases active surveillance network, 10 US sites, 2016-2019. Morb. Mortal. Wkly. Rep., 69(17): 509-514.

9. McLauchlin, J., Aird, H., Amar, C., Barker, C. and Dallman, T. (2020) Listeria monocytogenes in cooked chicken: Detection of an outbreak in the UK (2016-2017) and analysis of L. monocytogenes from unrelated monitoring of foods (2013-2017) Listeria monocytogenes and cooked chicken. J. Food Prot., 83(12): 2041-2052.

10. EFSA. (2017) The European Union summary report on trends and sources of zoonoses, zoonotic agents and foodborne outbreaks in 2016. EFSA J., 15(12): 5077.

11. Denny, J. and McLauchlin, J. (2008) Human Listeria monocytogenes infections in Europe-an opportunity for improved European surveillance. J. Eurosurveill., 13(13): 8082

12. EFSA, and Prevention, E.C.D. (2013) The European Union summary report on trends and sources of zoonoses, zoonotic agents and food-borne outbreaks in 2011. EFSA J., 11(4): 3129.

13. Odetokun, I.A. and Adetunji, V.O. (2016) Prevalence and persistence of Listeria monocytogenes in dairy and other ready-to-eat food products in Africa. In: Microbes in Food and Health. Springer, Berlin. p349-362.

14. Paudyal, N., Anihouvi, V., Hounhouigan, J., Matsheka, M.I., Sekwati-Monang, B., Amoa-Awua, W., Atter, A., Ackah, N.B., Mbugua, S. and Asagbra, A. (2017) Prevalence of foodborne pathogens in food from selected African countries a meta-analysis. Int. J. Food Microbiol., 249: 35-43.

15. Smith, A.M., Tau, N.P., Smouse, S.L., Allam, M., Ismail, A., Ramalwa, N.R., Disenyeng, B., Ngomane, M. and Thomas, J. (2019) Outbreak of Listeria monocytogenes in South Africa, 2017-2018: Laboratory activities and experiences associated with whole-genome sequencing analysis of isolates. Foodborne Pathog. Dis., 16(7): 524-530.

16. EFSA, Ricci, A., Allende, A., Bolton, D., Chemaly, M., Davies, R., Fernández Escámez, P.S., Girones, R., Herman, L. and Koutsoumanis, K.J.E. (2018) Listeria monocytogenes contamination of ready-to-eat foods and the risk for human health in the EU. EFSA J., 16(1): e05134.

17. Goutard, F.L., Bordier, M., Calba, C., Erlacher-Vindel, E., Góchez, D., de Balogh, K., Benigno, C., Kalpravidh, W., Roger, F. and Vong, S.J.B. (2017) Antimicrobial policy interventions in food animal production in South East Asia. BMJ, 358: j3544.

18. Addis, M. (2015) A review on antibiotic resistant and implication on food chain. J. Food Sci., 42: 9-11. 
19. Stratev, D. and Odeyemi, O.A. (2016) Antimicrobial resistance of Aeromonas hydrophila isolated from different food sources: A mini-review. J. Infect., 9(5): 535-544.

20. Regea, G. (2018) Review on antibiotics resistance and its economic impacts. J. Pharmacol. Clin. Res., 5(5): 555675.

21. de Noordhout, C.M., Devleesschauwer, B., Angulo, F.J., Verbeke, G., Haagsma, J., Kirk, M., Havelaar, A. and Speybroeck, N. (2014) The global burden of listeriosis: A systematic review and meta-analysis. Lancet Infect Dis., 14(11): 1073-1082.

22. Ranjbar, R. and Halaji, M. (2018) Epidemiology of Listeria monocytogenes prevalence in foods, animals and human origin from Iran: A systematic review and meta-analysis. BMC Public Health, 18(1): 1057.

23. Aureli, P., Ferrini, A.M., Mannoni, V., Hodzic, S., WedellWeergaard, C. and Oliva, B. (2003) Susceptibility of Listeria monocytogenes isolated from food in Italy to antibiotics. Int. J. Food Microbiol., 83(3): 325-330.

24. Byrne, V.D.V., Hofer, E., Vallim, D.C. and Almeida, R. (2016) Occurrence and antimicrobial resistance patterns of Listeria monocytogenes isolated from vegetables. Braz. J. Microbiol., 47(2): 438-443.

25. Castanon, J. (2007) History of the use of antibiotics as growth promoters in European poultry feeds. Poult. Sci. J., 86(11): 2466-2471.

26. Fakhri, Y., Rahmani, J., Oliveira, C.A.F., Franco, L.T., Corassin, C.H., Saba, S., Rafique, J. and Khaneghah, A.M. (2019) Aflatoxin M1 in human breast milk: A global systematic review, meta-analysis, and risk assessment study (Monte Carlo simulation). Trends Food Sci. Technol., 88: 333-342.

27. Cumpston, M., Li, T., Page, M.J., Chandler, J., Welch, V.A., Higgins, J.P. and Thomas, J. (2019) Updated guidance for trusted systematic reviews: A new edition of the Cochrane Handbook for Systematic Reviews of Interventions. Cochrane Database Syst. Rev., 10: ED000142.

28. Jordan, K. and McAuliffe, O. (2018) Listeria monocytogenes in foods. Adv. Food Nutr. Res., 86: 181-213.

29. Luber, P. (2011) The codex alimentarius guidelines on the application of general principles of food hygiene to the control of Listeria monocytogenes in ready-to-eat foods. Food Control, 22(9): 1482-1483.

30. Johansson, T. (1998) Enhanced detection and enumeration of Listeria monocytogenes from foodstuffs and food-processing environments. Int. J. Food Microbiol., 40(1-2): 77-85.

31. Donnelly C.W and Nyachuba D.G. (2007). Conventional methods to detect and isolate Listeria monocytogenes. In Listeria, Listeriosis, and Food Safety, $3^{\text {rd }}$ edn, pp. 215-249. Edited by E. T. Ryser \& E. H. Marth. Boca Raton, FL: CRC Press

32. Donnelly, C.W. and Nyachuba, D.G. (2007) Conventional methods to detect and isolate Listeria monocytogenes. $J$. Food Sci., 161: 215.

33. Cetinkaya, F., Elal Mus, T., Yibar, A., Guclu, N., Tavsanli, H. and Cibik, R. (2014) Prevalence, serotype identification by multiplex polymerase chain reaction and antimicrobial resistance patterns of Listeria monocytogenes isolated from retail foods. J Food Saf., 34: 42-49.

34. Osaili, T.M., Alaboudi, A.R. and Nesiar, E.A. (2011) Prevalence of Listeria spp. and antibiotic susceptibility of Listeria monocytogenes isolated from raw chicken and ready-to-eat chicken products in Jordan. Food Control, 22(3-4): 586-590.

35. Wong, W., Pui, C., Tunung, R., Cheah, Y., Nakaguchi, Y., Nishibuchi, M. and Son, R. (2012) Prevalence of Listeria monocytogenes in frozen burger patties in Malaysia. Int. Food Res. J., 19(4): 1751-1756.

36. Dalzini, E., Bernini, V., Bertasi, B., Daminelli, P., Losio, M.N. and Varisco, G. (2016) Survey of prevalence and seasonal variability of Listeria monocytogenes in raw cow milk from Northern Italy. Food Control, 60: 466-470.

37. Maas, C.J. and Hox, J. (2005) Sufficient sample sizes for multilevel modeling. Eur. J. Res. Methods Behav. Soc. Sci., 1(3): 86.

38. Allen, K.J., Wałecka-Zacharska, E., Chen, J.C., Katarzyna, K.P., Devlieghere, F., Van Meervenne, E., Osek, J., Wieczorek, K. and Bania, J. (2016) Listeria monocytogenes an examination of food chain factors potentially contributing to antimicrobial resistance. Int. J. Food Microbiol., 54: 178-189.

39. Tang, K.L., Caffrey, N.P., Nóbrega, D.B., Cork, S.C., Ronksley, P.E., Barkema, H.W., Polachek, A.J., Ganshorn, H., Sharma, N. and Kellner, J.D. (2017) Restricting the use of antibiotics in food-producing animals and its associations with antibiotic resistance in food-producing animals and human beings: A systematic review and meta-analysis. Lancet Planet. Health, 1(8): e316-e327.

40. Founou, L.L., Founou, R.C. and Essack, S.Y. (2016) Antibiotic resistance in the food chain: A developing country perspective. Front. Microbiol., 7: 1881.

41. World Health Organization. (2004) Joint FAO/OIE/WHO Expert Workshop on Non-Human Antimicrobial Usage and Antimicrobial Resistance: Scientific Assessment. World Health Organization, Geneva.

42. Heuer, O.E., Kruse, H., Grave, K., Collignon, P., Karunasagar, I. and Angulo, F.J. (2009) Human health consequences of use of antimicrobial agents in aquaculture. Clin. Infect. Dis., 49(8): 1248-1253.

43. Koluman, A. and Dikici, A. (2013) Antimicrobial resistance of emerging foodborne pathogens: Status quo and global trends. Crit. Rev. Microbiol., 39(1): 57-69.

44. Van, T.T.H., Yidana, Z., Smooker, P.M. and Coloe, P.J. (2020) Antibiotic use in food animals worldwide, with a focus on Africa: Pluses and minuses. J. Glob. Antimicrob. Resist., 20: 170-177.

45. Stonsaovapak, S. and Boonyaratanakornkit, M. (2010) Prevalence and antimicrobial resistance of Listeria species in food products in Bangkok, Thailand. J Food Saf., 30: 154-161.

46. Kovacevic, J., Mcintyre, L.F., Henderson, S.B. and Kosatsky, T. (2012) Occurrence and distribution of Listeria species in facilities producing ready-to-eat foods in British Columbia, Canada. J. Food Prot., 75(2): 216-224.

47. Yakubu, Y., Salihu, M., Faleke, O., Abubakar, M., Junaidu, A., Magaji, A., Gulumbe, M. and Aliyu, R. (2012) Prevalence and antibiotic susceptibility of $L$. monocytogenes in raw milk from cattle herds within Sokoto Metropolis, Nigeria. Sokoto J. Vet. Sci., 10(2): 13-17.

48. Olaimat, A.N., Al-Holy, M.A., Shahbaz, H.M., Al-Nabulsi, A.A., Abu Ghoush, M.H., Osaili, T.M., Ayyash, M.M. and Holley, R.A. (2018) Emergence of antibiotic resistance in Listeria monocytogenes isolated from food products: A comprehensive review. Compr. Rev. Food Sci. Food Saf., 17: 1277-1292.

49. Munita, J.M. and Arias, C.A. (2016) Mechanisms of Antibiotic Resistance. Wiley Online Library, Hoboken, New Jersey. p481-511.

50. Bashir, B., Manga, S., Kawo, A., Muazu, A., Danjuma, B., Ibrahim, M., Abdullahi, A. and Mawak, J. (2011) Susceptibility profile of Salmonella Typhi isolated from typhoid patients attending the Federal Medical Center, Gusau, Nigeria. Int. J. Biomed. Health Sci., 7(4): 265-268. 